\title{
Abstracts of Papers Read at the Meeting of the International Medical Society of Paraplegia, 1988
}

\author{
Held in Perth, Western Australia, May 1988
}

\author{
Thoracolumbar junction injuries: Do transitional variations influence level of \\ trauma? \\ K. P. Singer, MSc, MAPA, ${ }^{1}$ J. Willén, MD, ${ }^{2}$ P. D. Breidahl, MD, FRCR, FRACR ${ }^{3}$ \\ R. E. Day, $\mathrm{BE}^{4}$ \\ ${ }^{1}$ Department of Anatomy and Human Biology, The University of Western Australia, Ned- \\ lands 6009, 2 Spinal Injuries Unit, Royal Perth Rehabilitation Hospital, Shenton Park \\ 6008, ${ }^{3}$ Visiting Radiologist, Royal Perth Hospital, Perth 6000, ${ }^{4}$ Bioengineering Division, \\ Royal Perth Hospital, Perth 6000.
}

The often abrupt change in facet (zygapophyseal) joint orientation at the thoracolumbar junction, coupled with differences in segmental mobility appears to predispose the T10 to L2 segments to injury. It was speculated that most injuries involving rotation would be located at the transitional level, as experimental studies have indicated that sagitally oriented joints limit rotation. To determine whether any association exists between the transitional level and injury pattern, CT investigations of 36 spinal injured patients were studied. Burst fractures (Denis type $B[n=13]$ and Denis type A [n $=4]$ ) represented $47^{\circ} \%$ of all patients. Fourteen cases $\left(39^{\circ}\right.$ ) showed radiologic signs of a rotation injury with disruption to one or both zygapophyseal joints. This latter group included Denis type $\mathrm{D}$, burst/rotation injuries. The remainder showed relatively minor compression fractures with little displacement.

The level of the transition was plotted against the injury level for those with fracturedislocation/burst-rotation injuries and for the burst/compression fractures group. In the latter case there was a non-significant association between injury and transition $(r=$ $0 \cdot 01, p=0 \cdot 65$ ), however, the rotation injury group showed a stronger association between the transitional level and site of injury $(r=0.69, p=0.006)$. The rotation injury group was further characterised by greater variability in the level of transition and a high incidence of an abrupt change from coronal to sagittal orientation $\left(59^{\circ}{ }_{0}\right.$ burst vs $79 \%$ rotation group).

These trends suggest that variations in the level of transition at the thoracolumbar junction may influence the localising effects of rotation injuries. Other factors may be important in the determining the level(s) of burst/compression injuries.

A quantitative study of motoneurons and cortico-spinal fibres related to function in human spinal cord injury (SCI)

C. Kaelan, P. Jacobsen, P. Morling, B. A. Kakulas

Department of Neuropathology, Royal Perth Hospital and Neuromuscular Research Institute University of Western Australia, Perth, Western Australia.

Neurophysiological studies have suggested that transynaptic degeneration may occur in the motoneurons below the level of a traumatic lesion in SCI. In order to obtain anatomical confirmation of this possibility, anterior horn cells (AHC) in 4 patients with long standing clinically 'complete' SCI and in 2 patients with motoneurone disease (MND) were counted and compared with 4 normal controls. One thousand serial sections of the 
L3 segment were prepared and AHC on both the left and right sides were counted. The results were 6800,731 and 6121 on the left, and 6680,783 and 6432 on the right, being the mean number of AHC for the test cases, MND cases and normal controls respectively. Therefore no significant difference was found in the AHC population between the traumatic cases and the normal controls. There was, as would be expected, an $80^{\circ}{ }_{0}$ reduction in the number of motoneurons in patients with MND.

Because of electrophysiological evidence of continuity of long tracts in patients with 'complete' clinical SCI below the spinal lesion, it was decided to count the number of descending axonal fibres in patients with 'complete' and 'incomplete' paralysis. In 7 of 18 'complete' cases between 27 and 2619 central axonal fibres were found to have survived the injury. The remaining 11 had no central preserved axons. In 3 'incomplete' cases with motor function of between $2 / 5$ and $3 / 5$ on one side of their body the number of central fibres traversing the lesion, were 5708 (A69/107) being $14.87^{\circ}$ of of the estimated total for the cortico-spinal pathway, $6535\left(9 \cdot 87^{\circ}{ }_{0}\right)$ for A80/177 and 21978 $\left(36.69^{\circ}{ }_{0}\right.$ ) for $\mathrm{A} 80 / 244$. On the side of the body with no volitional control, the number of descending pyramidal tract fibres was $1214\left(3.5^{\circ}{ }_{0}\right)$ for A69/107, $3462(2.33 \%)$ for A80/177 and $2989\left(3.5^{\circ} \circ\right)$ for A80/244.

From this study the minimum number of central axons required to retain volitional movement of $2 / 5$ to $3 / 5$ was estimated to be between 3500 to 5500 , i.e. $3.5 \%$ to $10 \%$ of the total number of corticospinal fibres in man at T4. This finding is compared with that reported in animals $\left(5^{\circ}{ }_{0}\right)$ and in post chordotomy patients $\left(20^{\circ}{ }_{0}\right)$, and is the first such estimate in human SCI.

\section{The measurement of spasticity}

A. J. Douglas, G. H. Creasey, E. G. Walsh, P. Edmond Spinal Unit, Edenhall Hospital, Musselburgh, East Lothian, UK.

The concept of spasticity encompasses increased muscle tone and stretch reflexes, involuntary movements and clonus, and is difficult to quantify. A new system has been developed to measure muscle stiffness accurately and has allowed assessment of the effect of physiotherapy and of neuromuscular stimulation on skeletal muscles.

\section{Methods}

Thigh muscle stiffness was measured in patients with complete spinal cord injury. Known forces were applied to the horizontally placed lower limb using a printed torque motor. This resulted in movements of the lower leg. The position, velocity and frequency of limb oscillation and the e.m.g. activity associated with the motion were recorded and used to calculate the stiffness of the muscles involved (known as torque-induced motion analysis). Muscle stiffness measured in this way was related to clinical estimates of stiffness and was shown to be more objective and precise. Small differences could also be shown to be statistically significant. Thigh stiffness measurements were then made immediately before and after a period of rest, large passive movements similar to those applied by physiotherapists, or active contractions induced by electrical stimulation.

\section{Results}

A period of rest caused little change in paraplegic thigh muscle stiffness. However, the passive movements of spastic muscles, as carried out by physiotherapists, produced a quantifiable reduction both in intrinsic stiffness of muscle and in the e.m.g. activity associated with the stretch reflex. The repeated isotonic contractions induced by electrical stimulation produced an increase in muscle stiffness. Some paraplegics had trained quadriceps muscles from participating in a daily programme of electrical muscle stimulation. Their thigh muscle stiffness was not significantly different from that of untrained muscles. Stiffness of the trained muscles decreased immediately after passive movement and increased immediately following muscle stimulation, in a similar way to the untrained muscles. 


\title{
Conclusions
}

The development of a reproducible, quantitative measure of muscle stiffness will contribute to the assessment of all forms of treatment affecting spasticity.

\author{
Haemodynamic alterations in the paravertebral venous plexus following spinal \\ trauma \\ M. R. McClelland, V. N. Cassar-Pullicino, E. Calhoun, I. W. McCall, W. S. El Masri \\ Department of Spinal Injuries/Radiology, The Robert fones $E$ Agnes Hunt Orthopaedic \\ Hospital, Oswestry, Shropshire, UK.
}

Demonstration of the vertebral venous plexus in the absence of venous occlusion is possible by selective transfemoral vein catheterisation or intra-osseous spinal venography and is not seen during lower limb venography. Pedal intravenous injection of contrast medium for excretory urography in spinal injury patients demonstrated an alteration in the haemodynamic venous pathway.

Abnormal venous return via the paravertebral venous plexus in the absence of iliac or inferior vena-caval occlusion was shown in $73\left(70^{\circ}{ }_{0}\right)$ of 107 spinal injury patients studied prospectively. The venographic findings were compared with the appearances seen in 200 non-injured patients with normal lower limb venography utilising the same radiographic technique.

This preferential venous flow route is not related to the time interval after injury. It is demonstrable in the early as well as late post-traumatic period and is reproducible on subsequent examinations. Thirty three $\left(72^{\circ} \%\right.$ of 43 cervical injury patients and $40\left(62^{\circ}{ }_{0}\right)$ of 64 thoracolumbar injury patients showed this anomalous venous pathway.

It is more commonly seen in cervical injury (33 [72 $\left.{ }_{0}^{\circ}\right]$ of 43 patients) than thoracolumbar injury (40 [62\%] of 64 patients), and is closely related to the severity of neurological deficit occurring in $59\left(80^{\circ}{ }_{0}\right)$ of 75 patients with complete lesions.

The underlying pathogenic mechanisms and significance of such an altered venous pathway are discussed with special reference to the structural changes seen in the posttraumatic axial skeleton.

\section{Staffing the Spinal Injuries Unit \\ John D. Yeo \\ Royal North Shore Hospital, Sydney, Australia.}

The Spinal Injury Unit serving a population of 3 million should ideally contain 40 beds. The paraplegic casualty should have an average stay of 4 months and the tetraplegic 5 months. One hundred new admissions should be anticipated every year. The Unit should be situated in a large hospital with comprehensive Intensive Care and other consultant facilities. The Unit requires specially trained consultant staff to receive admissions within hours of the injury. Sufficient associated medical and auxillary medical staff are necessary to provide a 24 hour a day care for the acute and sub-acute patients. The Unit also requires to provide adequate rehabilitation services with separate physiotherapy, occupational therapy, treatment areas and hydrotherapy and sporting facilities. The patient should be introduced to vocational programmes with particular emphasis on the use of computers. Services from the orthotic consultant and department are essential. Appropriate secretarial assistants and ward clerks with computer facilities will allow adequate and easily retrieved patient data. There must be adequate staff to maintain at least 2 outpatient clinics per week with arrangements for the selected members of the Spinal Management Team to visit outlying hospitals in country and outer metropolitan areas 4 times a year. Patient and relative liaison nurses are essential to facilitate the transfer of the patient to the carers in the home environment. There must be adequate opportunities for discharged patients or their relatives to contact Unit staff urgently. The numbers of staff and the specialist staff required will be detailed with time tabling of combined staff activities and the mechanics of producing efficient referrals to other 
vocational training units for further medical and vocational rehabilitation. This same Unit should be involved in research. The importance of specially trained staff being involved in an awareness and prevention programme cannot be over emphasised. The success of this model in Australia should provide an incentive to other countries at present developing specialised Units for the treatment of patients with spinal cord injuries.

New spinal instrumentation for unstable thoracic and lumbar spine due to spinal tumour and fracture-dislocation

N. Tajima, K. Kawano, K. Matsumoto, K. Tashiro

Department of Orthopaedic Surgery, Miyazaki Medical College, Japan.

New spinal instrumentation (segmental square spinal instruments; 3-S) was produced as a trial and clinically applied to obtain stability for the unstable spine.

\section{Materials and methods}

New spinal instrumentation

The new system is a segmental square spinal instrumentation (termed 3-S) for posterior spinal fusion. It consists of pairs of transverse bars, hooks and longitudinal rods. The hooks were inserted into the lateral sides by inferior articular processes bilaterally and one pair of hooks were fixed by two transverse bars and a nut. Upper and lower hooks were linked by longitudinal rods.

\section{Clinical application}

Between 1984 and 1987, the instruments were used in 12 patients for spinal tumour and fracture-dislocation at the Miyazaki Medical College Hospital. The ages ranged from 2379 years. The mean follow-up period was 1 year and 4 months. The operation was combined with laminectomy, anterior spinal fusion and posterolateral spinal fusion in 10,2 and 4 patients, respectively. All the patients showed good stability post-operatively.

\section{Conclusion}

It has been demonstrated experimentally that new instrumentation yields a fixation force as firm as that of the conventional instrument, and this advantage was clinically confirmed. In the present trial, it was effective for patients with spinal tumours and fracture-dislocation in whom laminectomy was carried out together with the instrumentation. The stabilisation appears to be favourable for rehabilitation.

\section{Risk factors for heterotopic ossification in spinal cord injured \\ S. Lal, A. Heinemann, B. Hamilton \\ Rehabilitation Institute of Chicago, Chicago, USA.}

Spinal cord injured (SCI) Patients have multiple complications in addition to the paralysis. Heterotopic Ossification ( $\mathrm{HO})$ is a complication in $16-53^{\circ}{ }_{0}$ of spinal cord injured patients. One third of these patients have moderate to severe $\mathrm{HO}$ that adversely affects their function or health. Pharmacological prophylaxis of HO for all SCI patients continues to be controversial. High risk criteria for $\mathrm{HO}$ formation identified in total hip replacement patients are not applicable to SCI patients. A review of the literature did not reveal specific factors for HO with SCI. The charts of 100 SCI patients, 50 with $\mathrm{HO}$ and 50 without $\mathrm{HO}$ were reviewed retrospectively to learn if criteria which would predict high risk patients could be identified. A total of 14 variables; 7 demographic (age, sex, race, level and completeness, cause of injury, and address state of patient), and 7 medical (bladder stones, fractures, pressure ulcers, deep vein thrombosis, pulmonary embolism, spasticity and urinary tract infections) were studied. Four of the 14 variables (age, completeness of injury, presence of pressure sores and spasticity) were significantly 
related to $\mathrm{HO}$ formation. The risk factors appear to be additive. When all were present, $92 \%$ of patients had HO. It is suggested that prophylaxis may be provided in the high risk group with one or more risk factors. A prospective study is recommended to varify the relationship of these high risk factors with $\mathrm{HO}$ formation in SCI patients.

\section{Spinal cord injuries and anejaculation}

J. A. Beilby, E. J. Keogh

The Reproductive Medicine Research Institute (Inc.), QEII Medical Centre, Nedlands, W.A. Australia 6009.

Since 1983, 41 men with spinal cord injury (SCI) have been referred to the Institute for evaluation and treatment of anejaculation and infertility. Ten men had lesions below T12 and 31 men had lesions above T11. Electroejaculation was performed on 12 men using the O'Leary electroejaculator and vibroejaculation was attempted in 37 men using a PIFCO 1558 vibratory massager which had been modified to give an amplitude of 2-4 $\mathrm{mm}$ when applied to the glans penis ( $8 \mathrm{men}$ had both techniques). The time taken to achieve ejaculation was recorded and routine semen analysis was performed on all ejaculates. Repeated ejaculation ( $\mathrm{n}>5$ ) has been at 1-3 week intervals in 9 men to date. Electroejaculation was successful on at least one occasion in 3 of 4 men with SCI below T12 and in 5 of 8 men with SCI above T11. Semen volume was low and sperm motility and did not improve with repeated ejaculation.

The modified PIFCO vibrator failed to induce ejaculation on at least 2 attempts per person in 9 men with SCI below T12. For men with SCI above T11, the vibrator induced ejaculation in 19 of 28 men $\left(68^{\circ}{ }_{0}\right)$. To date 99 ejaculations have been obtained from 125 attempts in these 19 men. Using a modification to the Life Table Analysis Technique, the time to achieve an ejaculation rate of $50^{\circ}{ }_{0}$ ranged from 35 seconds to 16 minutes and the peak ejaculation rate ranged from $62 \cdot 5^{\circ}{ }_{0}$ to $100^{\circ}{ }_{0}$. Semen volume was normal, and motility although low was significantly better than that from electroejaculation. Repeated ejaculation led to improved semen quality in all patients even though the average time from injury to first semen collection was 7.8 years (range $1.5-20$ years).

The vibrator causes true ejaculation in contrast to electrical stimulation which at best led to semen trickling from the meatus and it usually required prostatic and urethral massage to obtain any fluid. In our hands electroejaculation did not induce hypertension, however this was seen prior to, during and following vibroejaculation. On one occasion this warranted abandoning the procedure.

In conclusion, electroejaculation consistently gave poor quality semen. Application of the vibrator produced ejaculation in $68^{\circ}$ o of men with SCI above T11. Provided that adequate measures are available to relieve hypertension, then this simple modification to a cheap, readily available massager, will mean that many more men with anejaculation due to SCI will be able to collect semen in the privacy of their own homes.

\section{Burst fractures in the thoracic and lumbar spine; a clinico-neuropathological analysis \\ J. Willen, U. Gaekwad, B. Kakulas \\ Department of Neuropathology, Royal Perth Hospital, Box X2213, GPO Perth, Western Australia 6001.}

Neuropathological analysis of 8 acute and 12 chronic burst fractures was performed. In the acute cases the injury to the bony, ligamentous and neural tissues was investigated.

Serious Denis B or D fractures showed signs of pronounced instability and all had a large bone fragment rotated into the spinal canal. The neural tissues were compressed in 2 cases and transected in 1.

The Denis A fractures had relatively well restored ligamentous structures and the bone fragment in the spinal canal was narrowing the spinal canal less than $50^{\circ}{ }_{0}$ in all cases. Three of 4 had normal neural tissue macro and microscopically.

The majority of the patients with chronic burst fractures did not show any sign of bone fragment resorption. 
Six out of 8 patients with thoracolumbar (T12 and L1) and lumbar (L3) fractures experienced intractable burning pain and/or rhizopathy. The pain seemed to be caused by entrapment of the nerve roots in adhesions.

\section{Microangiographic study of spinal cord injuries in man: Traumatic lesion versus myelopathy \\ H. Shingu, MD, I. Kimura, MD, Y. Nasu, MD, A. Shiotani, MD, M. Oh-hama, MD \\ Department of Orthopedic Surgery, San-in Rosai Hospital, Kaike-Shinden, Yonago-si, 683, fapan.}

Postmortem microangiography has been performed in 12 cases with traumatic cervical cord injury, 2 cases with myelopathy and 1 case with post-traumatic syringomyelia.

In traumatic lesions, the size and extent of the non-filling areas was directly related to the injury force and length of time elapsed following the injury. Subsequently the nonfilling areas became cavitated. In myelopathy, the hypervascular areas were observed just surrounding the chronic compressed area. In post-traumatic syringomyelia, the capillaries in the posterior horns had disappeared, and the central arteries were distorted by distending forces of the syrinx. The microangiographs suggested that vascular factors might play a significant role in the original cavity formation; but in cavity extension, these factors were not primary.

The effect of early administration of scopolamine on spinal cord injury: An experimental study

S. Shao-ting, X. Zhong-guang, J. Lu-lin et al.

General Hospital of Beijing Unit of People's Liberation Army, China.

Scopolamine was given IM 20 minutes after experimental spinal cord injury caused by 545 GCM in 64 dogs. Six weeks later, the recovery rate in 11 dogs treated with large doses of scopolamine was $54.5 \%$ and $36.3^{\circ}{ }_{0}$ of the animals were able to walk. In the group that received the smaller doses of scopolamine, $81.8 \%$ recovered and $45.3 \%$ returned to walk. In the control group of 13 dogs, none recovered. Data of SEP and PSR determination were in close correlation with the results of the neurological status. Microscopically, there was normal white matter in the periphery and also some corners of grey matter preserved in the specimens obtained in the dogs that recovered walking ability, while in the untreated dogs, the whole segment of the traumatised spinal cord was replaced by glial cells and fibrous tissue. Under the electron microscope, the findings were: obstruction of the capillaries by RBC and platelets in the injured segment of the untreated dogs and normal capillaries in the specimens of the treated group. Obviously, the effect of scopolamine was to regulate the microcirculation of the traumatised spinal cord. 